\title{
Influence of Backscattered Electron Imaging Geometry on Channeling Contrast of Dislocations
}

\author{
Ranga J. Kamaladasa and Yoosuf N. Picard \\ Materials Science and Engineering, Carnegie Mellon University, Pittsburgh, PA 15213
}

Backscattered electron (BSE) imaging is conventionally utilized for enhanced topographic and compositional imaging in scanning electron microscopy (SEM). With broad availability of high efficient solid state detectors and high brightness field emission guns, electron channeling contrast imaging (ECCI) has become a growing technique for non-destructive imaging of crystalline orientation variations, strain fields and extended defects. Recent studies have implemented ECCI using commercial forescatter diode detectors [1-2] and backscatter diode detectors [3] for dislocation analysis. The incident angular trajectory differences between backscatter and forescatter geometries each afford the microscopist certain advantages in utilizing ECCI. However, the channeling contrast behavior of specific dislocations under different imaging geometries and SEM conditions is still not well understood. Also, more conventional methods for dislocation analysis, such as transmission electron microscopy (TEM) or defect-selective chemical etching, have not been widely utilized to verify dislocation imaging/identification by ECCI.

In this study, we implement ECCI in both forescatter and backscatter geometries under various SEM conditions (e-beam voltage and current) for imaging dislocations in $\mathrm{GaN}$ and $\mathrm{SrTiO}_{3}$. For GaN, ECCI is found to resolve edge and screw threading dislocations by monitoring the spatial size difference of the contrast features, a result confirmed by coordinated site-specific chemical etching and TEM analysis. Additionally, the diffraction contrast of screw component dislocations is found to behave similarly for ECCI and TEM under different diffraction conditions. Invisibility criterion $(\mathbf{g} \cdot \mathbf{b}$ and $\mathbf{g} \cdot \mathbf{b x u})$ is demonstrated for sub-surface dislocations in $\mathrm{SrTiO}_{3}$. Other sub-surface defects, such as dislocation loops [Figure 1], have continuous changes to the local dislocation line direction that yield continuous modulations in BSE intensity under various diffraction conditions.

Since the backscatter geometry allows efficient BSE collection over a wider angular tilt range than the forescatter geometry, we find the backscatter geometry advantageous for ECCI where many specific diffraction conditions must be accessed. However, we also find benefits in utilizing forescatter geometries in order to access out-of-plane diffraction conditions necessary for more robust invisibility criterion analysis. Also, edge and screw dislocations show less spatial size differences when imaged by ECCI using forescatter diodes, a possible consequence of enhanced surface sensitivity to the near surface strain fields surrounding edge dislocations [Figure 2].

The techniques of dislocation-selective etching and ECCI have been compared over large areas of GaN and $\mathrm{SrTiO}_{3}$ substrates, showing excellent 1:1 correlations for surface penetrating dislocations spanning densities from $10^{5}-10^{8} / \mathrm{cm}^{2}$. The coordinated etching and ECCI studies of $\mathrm{SrTiO}_{3}$ have revealed the nature of contrast features for inclined dislocations and the configuration of dislocation half-loops. These results further establish ECCI as a promising technique for the microscopist already equipped to perform conventional BSE imaging. 


\section{References}

[1] C. Trager-Cowan, S. K. Manson-Smith, D. A. Cowan, F. Sweeney, D. McColl, A. Mohammed, R. Timm, P. G. Middleton, K. P. O’Donnell, D. Zubia, and S. D. Hersee, Mater. Sci. Eng., B $82(2003) 19$.

[2] Y. N. Picard, M. E. Twigg, J. Appl. Phys. 104 (2008) 124906.

[3] M.A. Crimp, Micro. Res. Tech. 69 (2006) 374.

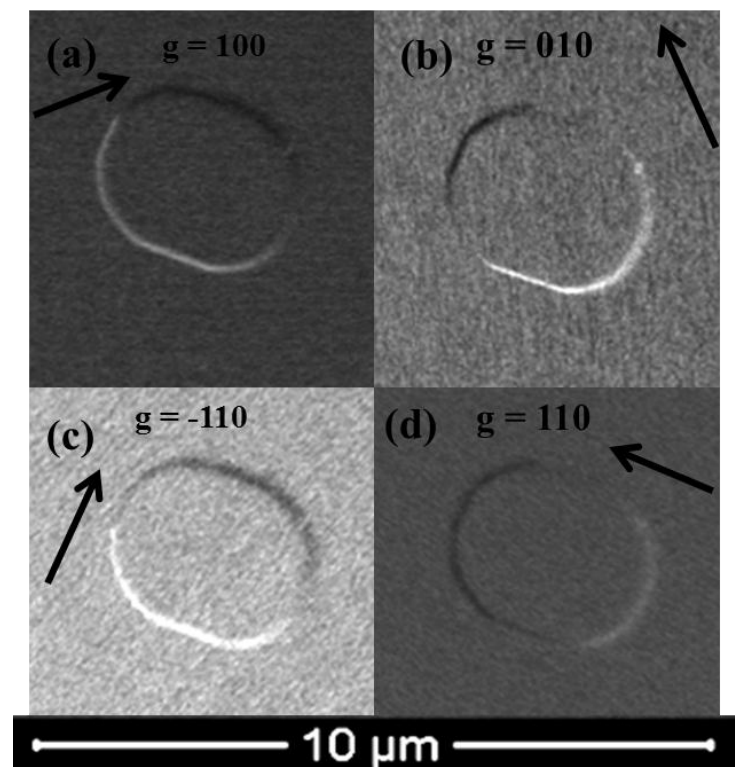

FIG. 1. Dislocation loop in $\mathrm{SrTiO}_{3}$ imaged under four different diffraction conditions using a polepiece mounted backscatter detector.
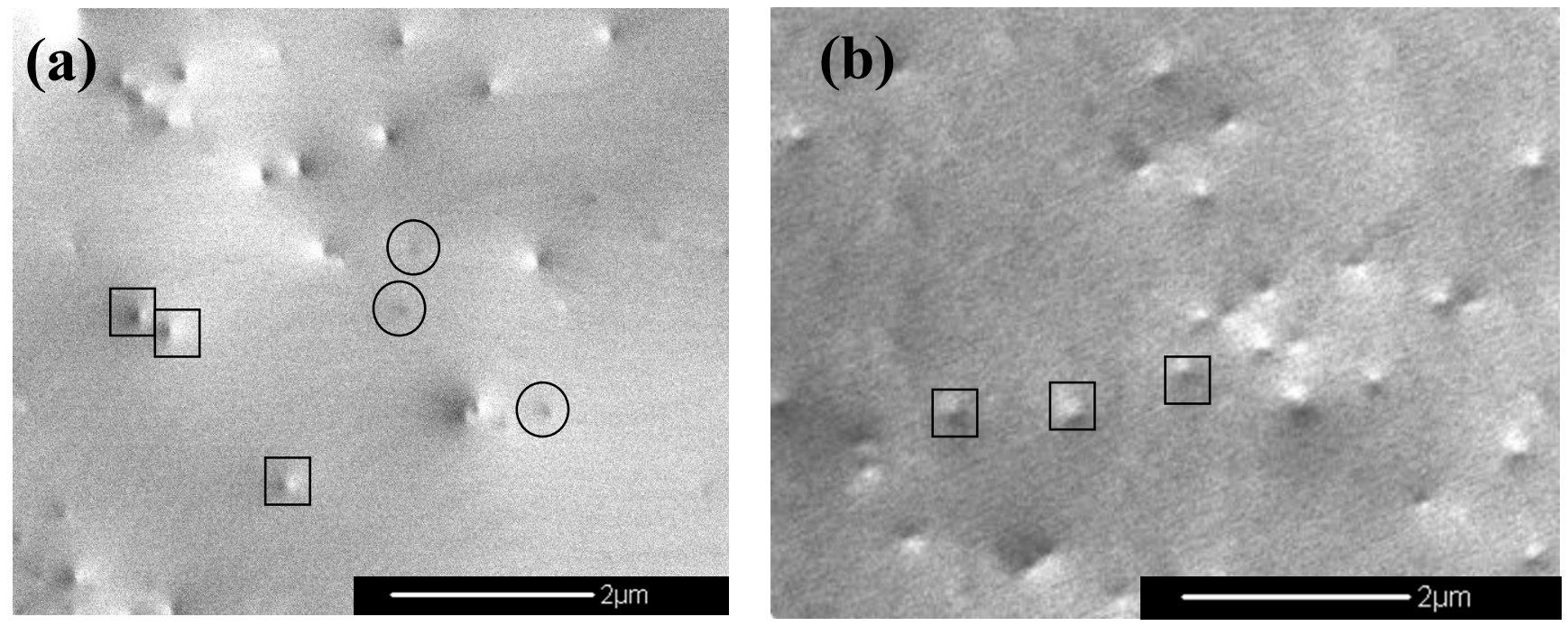

FIG. 2. ECCI micrographs of MOCVD grown GaN (0002) film imaged by (a) forescatter diodes and (b) backscatter diodes. Edge dislocations are denoted by circles while screw dislocations are denoted by squares. 